\title{
Prendre langue
}

Pierre Lexert

\section{(2) OpenEdition}

Journals

Édition électronique

URL : http://journals.openedition.org/esp/440

DOI : $10.4000 /$ esp.440

ISSN : 2532-0319

\section{Éditeur}

Centre d'Information sur l'Éducation Bilingue et Plurilingue

\section{Édition imprimée}

Date de publication : 1 décembre 2014

Pagination : 85-88

ISSN : 1127-266X

\section{Référence électronique}

Pierre Lexert, «Prendre langue », Éducation et sociétés plurilingues [En ligne], 37 | 2014, mis en ligne le 01 octobre 2015, consulté le 14 septembre 2020. URL : http://journals.openedition.org/esp/440 


\section{«PRENDRE LANGUE»}

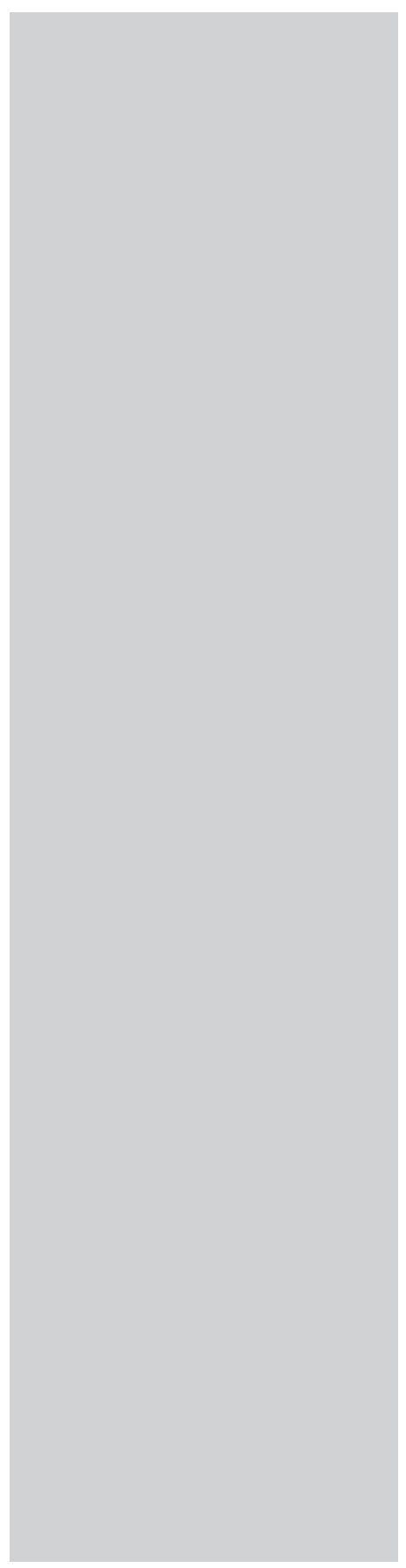

\section{Pierre LeXerT}

Q

uelle jolie, rapide et pertinente façon de signifier une accointance avec autrui que l'expression: prendre langue!

Aussi bien implique-t-elle d'abord un "apprendre langue". La langue, instrument magique de la communication, de l'échange, de la confidence, du savoir, des rapports humains, du biendire... D'où la nécessité ou la tentation d'en partager les divers codes si besoin, soit en s'y préparant, soit en se pliant aux circonstances.

C'est à ce dernier concours - de circonstances - que je dus de m'approprier plus ou moins bien quatre parlers, et du passé et du présent. Du passé, pour ce qui est du dialecte valdôtain, branche du mal nommé francoprovençal, qui fut, avec le français, le lait mental de ma prime enfance. Patois très ancien - proto-français de l'aire lyonnaise (Gaston Tuaillon dixit) - employé par les paysans de la commune de Fénis, en Vallée d'Aoste, dont étaient originaires mes géniteurs; et où je fus élevé jusqu'à ma cinquième année par une tante et ma grand'mère durant que mes parents, émigrés, se donnaient à faire en France. Ce patois en effet, émanait d'un milieu essentiellement agricole dont les us et moyens n'avaient guère varié depuis le moyen-âge, étant pratiquement restés en l'état. Heureusement, il se doublait de son avatar autrement plus évolué et policé: le français contemporain, avec lequel nos paysans s'étaient parallèlement familiarisés, par le biais des spectacles itinérants, foires et manifestations auliques, et leurs rapports avec les notaires, les clercs, les avocats, les médecins, les géomètres, les colporteurs.

Quant au présent, outre le français, l'anglais et l'allemand comptaient parmi les langues des grandes puissances occidentales et allaient donc bientôt solliciter mon attention. Mais c'est ma polyvalence intellectuelle qui me valut d'être d'abord contraint de taquiner la Lorelei germanique. Voici pourquoi: j’avais accumulé les prix d'excellence à l'école communale du IVè arrondissement de Paris (ayant entre temps rejoint père et mère) et même rem- 
«Prendre langue»

P. LEXERT porté le premier prix de cet arrondissement dans ma onzième année pour une rédaction sur les méfaits de l'alcoolisme. Car mon français ne souffrait pas de mes enfances valdôtaines, pratiqué qu'il était de fort classique manière, au sein de notre foyer, sous l'égide d'un grand-père, ex-maréchal des carabiniers puis syndic de la commune.

De sorte que, sorti premier du concours d'entrée au Lycée Charlemagne, je fus l'objet de la sollicitude du proviseur, qui, bien que me sachant désireux d'apprendre l'anglais, me suggéra "fermement" de m'appliquer prioritairement à l'allemand parce qu'il estimait que cette langue, du fait de ses déclinaisons, était aussi formatrice que le latin à peu de chose près.

Je rechignais assez à cette injonction bénévolente, qui contrariait les idées reçues de ma juvénile ignorance, et c'est plutôt mal engroin que j'assistais aux leçons d'un brave germaniste blanchi sous le harnais mais totalement dénué de charisme. Ainsi traînaisje deux ans en queue du peloton, avant que n'intervint un professeur de souche autrichienne qui sut me vanter les charmes de la poésie d'outre-Rhin. Au point que, ayant cru avoir bâclé une composition mensuelle consistant à traduire un sermon de Luther - incroyant que j'étais déjà ! - j’appris avec stupéfaction que ma version, non seulement était la meilleure, mais que le professeur déclarait qu'il n'aurait pas pu faire mieux!

Et sans doute ma connaissance de l'allemand m'a-t-elle sauvé la vie, alors que pourchassé par les nazis après m'être évadé d'un faux maquis landais durant l'Occupation de la France, je parvins in extremis à faire s'arrêter un camion de la Kommandantur de Mont-de-Marsan, et dialoguant avec le conducteur, le persuader faussement que j'avais dessein de m'y rendre. Toujours est-il que je me suis plus aisément accommodé de la prononciation de l'allemand - en adoptant même la calligraphie gothique - que de celle de l'anglais, auquel je me frottai dès la classe de 4è, trois ans avant le baccalauréat.

M'y portait la faveur dont il bénéficiait déjà et mon intérêt ou mon admiration pour Walter Scott, Jack London, Fenimore Cooper, le Defoe de Moll flanders... et Fred Astaire, l'incomparable danseur de claquettes, dont nous fredonnions les chansons. (Il fut cause que, quelque quinze ans plus tard, je pris des cours de claquettes, lesquels, avec les séances d'escrime, me tenaient lieu de sports hebdomadaires).

Si je n'ai guère eu l'occasion par la suite de tirer parti de ma connaissance de l'allemand - malgré de brèves incursions en Alsace, en Suisse alémanique, en Basse-Saxe, en Bade- 
«Prendre langue»

P. LEXERT
Wurtemberg et en Autriche, qui ont compté pour du beurre - en revanche, l'anglais me devint plus familier lorsque, Directeur du département des Recherches de Publicis - actuellement la plus importante multinationale publicitaire du monde - il me fallut m'instruire en matière de sondages d'opinion, en organiser, en exploiter, et assister à des congrès y afférents sur lesquels planait l'ombre américaine de Gallup qui en fut le grand instigateur et théoricien. L'acquisition et la maîtrise d'un vocabulaire technique, une fois assimilés les rudiments généraux, pose en effet peu de problèmes. D'autant que, parallèlement, mon goût pour les belles-lettres, m'inclinait à lire et parfois traduire: Edgar Poe, Hawthorne, Wilkie Collins, Oscar Wilde, Chesterton, Rex Warner, et surtout la fascinante et trop peu louée Anthologie de Spoon River d'Edgar Lee Masters.

Devenu ensuite Président Directeur Général de la société Matière E Maîtrise, je fus amené à me rendre à Londres, à la Foire du "Do it yourself", et à y négocier un procédé de fabrication avec un Anglais, si typique sujet de la dite "perfide Albion" (laquelle en compte aussi de fort estimables) qu'il se révéla peu après aussi arrogant que malhonnête. Ce qui nourrit entre nous des échanges acidulés et m'engagea à entreprendre un bénéfique voyage en Hollande - y consulter L'Institut international des Brevets - à l'issue duquel je pus lui river son clou et l'envoyer paître.

Enfin, vers 1980, je parvins à me mettre en rapport avec un écrivain britannique, Rex Warner, qui avait précédemment retenu mon attention. Il professait les humanités à Oxford, mais était aussi l'auteur, outre un essai sur Périclès, de deux remarquables romans: L'Aérodrome et La Chasse à l'oie sauvage. De là, entre nous, une sympathique correspondance (il m'écrivait en anglais), dont j'ai fait relier des spécimens avec deux éditions originales de ses romans.

Pour en revenir à la prononciation de l'anglais, il est intéressant de relever qu'il résulte des observations et recherches d'Alfred Tomatis (oto-rhino-laryngologiste de réputation mondiale, Français de souche italo-niçoise) que les autochtones de France et d'Italie sont les bien moins armés parmi les Européens pour s'exprimer oralement dans la langue d'Oscar Wilde. Et cela du fait de leurs facultés acoustiques, dépendantes de leur environnement géophysique. Ce que nos piètres performances dans ce domaine ne peuvent que confirmer, sans même tenir compte des ségrégations que provoque leur façon de parler parmi les citoyens d'outre-Manche.

Et puis... Et puis... Les années passant, et compte tenu de divers 
«Prendre langue»

P. LEXERT facteurs domestiques, je décidai, bien qu'encore vert, de retourner dans mon Val d'Aoste, afin d'y cultiver le domaine familial et donner libre cours à ma vocation littéraire. Laquelle fut encouragée et favorisée par l'un des plus grands libraires de France ainsi que par mes fonctions de Directeur de l'Institut valdôtain de la Culture (IVAC) et de rédacteur en chef des Cahiers $d u R u$, que 48 numéros imposèrent comme l'une des revues phares de l'Espace francophone.

Ce qui me donna tout loisir, en composant mon œuvre, de prendre encore mieux conscience au long aller de la miraculeuse ductilité du français - en comparaison duquel l'anglais me semble moins précis et l'allemand plus pesant. Il en résulte que, n'ayant plus guère l'occasion de les pratiquer, sauf incidemment, il ne m'en reste en mémoire que quelques bribes; bribes que je triture laborieusement si besoin est, mais qu'il me suffirait de la perspective d'une plus longue vie pour entreprendre de les étoffer à nouveau. 